\title{
Adherencia al Tratamiento basado en Comportamientos en Pacientes Diabéticos Cartagena de Indias, Colombia
}

\author{
A treatment-adherence study based on diabetic patients' behaviour in \\ Cartagena, Colombia
}

Alicia N. Alayón ${ }^{1}$ y Mario Mosquera-Vásquez ${ }^{2}$

1 Programa de Bacteriología de la Facultad de Ciencias de la Salud. Universidad de San Buenaventura. Cartagena, Colombia. alinorala@hotmail.com, alinorala@gmil.com

2 Departamento de Comunicación Social, Universidad del Norte. Barranquilla, Colombia

Recibido 9 Abril 2008/Enviado para Modificación 19 Octubre 2008/Aceptado 9 Noviembre 2008

\section{RESUMEN}

Objetivo Conocer los niveles de adherencia auto-reportada y control metabólico, interpretados desde un enfoque biopsicosocial, con el fin de proponer estrategias culturalmente adaptadas que permitan el logro de mejores resultados en los programas impartidos.

Métodos se realizó un estudio descriptivo que incluyó 131 pacientes diabéticos usuarios de una empresa promotora de salud (EPS) de la ciudad de Cartagena de Indias, Colombia. Se aplicaron entrevistas para conocer los niveles de adherencia auto-reportada y se midió hemoglobina glucosilada A1C para evaluar control metabólico.

Resultados En una escala de 0 a 7 , el puntaje promedio de adherencia obtenido fue de 4,6 (IC $95 \% 3,8-5,4)$. Los mayores puntajes fueron 6,6 para no tabaquismo (IC $95 \% 5,5-7,8)$ y 6,2 para medicación (IC $95 \%$ 5,9-6,6). Los menores fueron 0,4 para automonitoreo (IC $95 \%$ 0,4-0,5); 3,4 para ejercicio físico (IC $95 \%$ 2,8-4,0) y 4,4 para consumo de vegetales (IC $95 \% 3,7-5,2$ ). El control metabólico deseable se logró en el $58 \%$ de los pacientes, pero no guardó relación con la adherencia auto-reportada ( $p>0,05)$.

Conclusiones Se recomienda evaluar la adherencia de manera independiente para cada comportamiento, fortalecer los pilares no farmacológicos del tratamiento en los programas dirigidos a pacientes diabéticos e implementar las acciones pertinentes para lograr mayores niveles de accesibilidad a éstos.

Palabras Clave: Diabetes mellitus, educación en salud, cooperación del paciente, enfermedad crónica (fuente: DeCS, BIREME).

\section{ABSTRACT}

Objective Ascertaining levels of self-reported adherence and metabolic control (from a bio-psychosocial perspective) aimed at putting forward culturally-sensitive 
strategies leading to better results being achieved by diabetes programmes.

Methods A descriptive study was conducted, involving 131 diabetic patients who were affiliated to one of the largest health insuring entities in the city of Cartagena, Colombia. Interviews were held for ascertaining the levels of self-reported adherence; glucosylated haemoglobin A1c was measured to evaluate metabolic control.

Results The average adherence score obtained on a scale from 0 to 7 was of 4,6 $(3,8-5,495 \% \mathrm{Cl})$. The highest scores were 6.6 for non-tobacco use $(5,5-7,895$ $\% \mathrm{Cl})$ and $6, .2$ for medication $(5,9-6,695 \% \mathrm{Cl})$. The lowest ones were 0.4 for selfmonitoring $(0,4-0,595 \% \mathrm{Cl}), 3,4$ for physical exercise $(2,8-4,095 \% \mathrm{Cl})$ and 4,4 for consuming vegetables $(3,7-5,295 \% \mathrm{Cl})$. The desired metabolic control was achieved in $58 \%$ of patients; however, these results were not consistent with selfreported adherence.

Conclusions The authors recommend independently evaluating adherence for each pattern to strengthen non-pharmacological treatment as part of diabetes' programmes and to implement relevant action for achieving greater accessibility to such programmes.

Key Words: Diabetes mellitus, health education, patient compliance, chronic disease (source: MeSH, NLM).

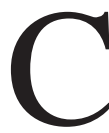
artagena de Indias es la capital de Bolívar, con una población de 1069 755 habitantes; concentrada principalmente en la zona urbana (92,5 \%). El 47,8 \% de los habitantes son hombres y el 52,2 \% mujeres (1).

La Ley 100 de 1993 lleva a Colombia a ser el primer país de ingresos medios que adoptó un modelo de competencia estructurada o mercado regulado para reformar el sistema de salud $(2,3)$.

En este sistema están previstos dos tipos de regímenes de afiliación: el contributivo y el subsidiado. Dentro del primero se crearon las empresas promotoras de salud (EPS) que en Cartagena de Indias suman en este momento un total de 13 (4), las cuales buscan mejorar y/o mantener la salud y la calidad de vida de sus afiliados (5).

Por su elevada frecuencia, la velocidad del incremento en el número de personas afectadas y sus implicaciones socioeconómicas, la diabetes mellitus es considerada un problema de salud pública (6). En 1995 había alrededor de 135 millones de diabéticos en el mundo, y se espera que, especialmente en países en vía de desarrollo, esta cifra se eleve a 300 millones en el 2025 (7).

En Colombia, las estadísticas para el departamento de Bolívar muestran a la diabetes como la décima causa de mortalidad, con una tasa de 2,3 por cada 
100.000 habitantes (8). En Cartagena de Indias, específicamente, su prevalencia es de 8,9 \% en mayores de 30 años (9), y es la novena causa de muerte en la población general; por ello se ha decidido considerar a la diabetes como una de las enfermedades cardiovasculares, y darle prioridad en los programas de acción de los próximos años $(10,11)$.

Estos programas, en el paciente diabético, están dirigidos a inducir y mantener en el tiempo una serie de cambios comportamentales para sostener sus niveles de glicemia lo más cercano posible a la normalidad, modificando de manera importante la historia natural de la enfermedad (12-14).

De lo anterior, surge el concepto de adherencia, entendido como la acción de involucrarse activa y voluntariamente en el manejo de la enfermedad, mediante el desarrollo de un proceso de responsabilidad compartida entre el paciente y los profesionales de la entidad prestadora de servicios en salud (15), que exige miradas que revaloricen lo subjetivo (16).

En los países en desarrollo el promedio de la adherencia a los tratamientos a largo plazo alcanza sólo el 50 \%, siendo incluso menor en los países en vías de desarrollo (17). En promedio, sólo uno de cada tres pacientes sigue de manera correcta las indicaciones de su médico (18), con adherencias diferentes para cada componente, mayores para la toma de medicamentos, aun en casos de polifarmacia $(19,20)$ y menores para ejercicio y dieta $(21)$.

Por otra parte, la falta de adherencia tiene repercusiones económicas. En Estados Unidos, para el 2002, los gastos en salud de las personas con diabetes se calculaban cercanos a los 132 billones de dólares, más del doble que de los no diabéticos (22). España reportó, para ese mismo año, un gasto de 883 euros en pacientes sin complicaciones, 1403 euros en aquellos con complicaciones microvasculares y más de 2000 euros en las de causas macrovasculares o mixtas (23-25).

Sin embargo, muchos programas educativos tendientes al mejoramiento de la adherencia se desarrollan de espaldas al tipo y grado de dificultad específica que enfrenta el grupo al que van dirigidos.

\section{MÉTODOS}

Se realizó un estudio descriptivo de corte transversal, durante el 2006, que incluyó la totalidad de los pacientes diabéticos mayores de 30 años $(\mathrm{N}=131)$ de 
una empresa promotora de salud (EPS) en Cartagena de Indias, Colombia, para asegurar que tuvieran acceso a programas de promoción y prevención y poder valorar la adherencia al tratamiento. El objetivo del estudio consistió en conocer los niveles de adherencia auto-reportada y control metabólico, interpretados desde un enfoque biopsicosocial, con el fin de proponer estrategias culturalmente adaptadas que permitan el mejoramiento de los programas que se imparten.

Para evaluar la adherencia se utilizaron entrevistas estructuradas a partir de la adaptación de una escala cuantitativa desarrollada por Toobert (26), que evalúa el cumplimiento de las recomendaciones, en los siete días previos, respecto a los cinco elementos fundamentales del cuidado de la diabetes: dieta, ejercicio, automonitoreo de glicemia, cuidado de pies y tabaquismo.

Se escogió el método del auto-reporte porque ha sido aceptado ampliamente como uno de los mejores (19) para evaluar la adherencia al tratamiento y se ha demostrado que es incluso mejor que métodos objetivos tales como el recuento de medicamentos remanentes (27). La escala fue sometida a pruebas pilotos; revisada y avalada por un experto en psicología y otro en el área de la comunicación en salud.

El control metabólico fue evaluado midiendo la concentración de hemoglobina glucosilada A1C (HbA1C), para lo cual se utilizó un equipo Nycocard Reader II, basado en cromatografía de afinidad por boronato, con controles de calidad de niveles alto, medio y bajo. Los resultados de las entrevistas y procedimientos bioquímicos fueron procesados en SPSS versión 13.0 y en Excel.

Los comités de ética de las universidades del Norte, de Barranquilla, y de San Buenaventura, sede Cartagena de Indias, a las que pertenecen los autores, estudiaron y aprobaron el protocolo del presente trabajo. Los pacientes incluidos en la investigación firmaron, de manera libre y voluntaria, una cláusula de consentimiento informado, que se elaboró según la Resolución 8430/93 del Ministerio de Salud de la República de Colombia. Las identidades de estos pacientes se mantuvieron en reserva; además, se les aseguró el acceso permanente a toda la información requerida. 


\section{RESULTADOS}

\section{Adherencia auto-reportada}

La muestra quedó conformada por 73 mujeres y 58 hombres para una distribución porcentual de 56 y $44 \%$ respectivamente, con una edad promedio general de 60,5 años y una desviación estándar de 11,3 años. Al momento de ser incluidos en el estudio el total de la población se encontraba participando activamente en los programas de seguimiento y control que brinda la entidad.

La escala aplicada permitió evaluar la adherencia auto-reportada para cada acción específica, de tal manera que un puntaje de 7 correspondió a adherencia perfecta y un puntaje de 0 correspondió a ausencia total de ésta. Los resultados obtenidos se muestran en la Tabla 1.

Tabla 1 Puntuación de adherencia auto-reportada para

\begin{tabular}{lcc}
\multicolumn{3}{c}{ cada comportamiento evaluado } \\
\hline Adherencia a: & $\begin{array}{c}\text { Puntuación } \\
\text { Obtenida } \\
\text { (Promedio) }\end{array}$ & $\begin{array}{c}\text { Intervalo de Confianza } \\
\text { (95\%) }\end{array}$ \\
\hline Medicación & 6,2 & $5,9-6,6$ \\
Consumo de vegetales & 4,4 & $3,7-5,2$ \\
Dieta sin grasas & 5,6 & $4,6-6,5$ \\
Dieta sin dulces & 5,9 & $4,9-7,0$ \\
Ejercicio físico & 3,4 & $2,8-4,0$ \\
Automonitoreo de glucemia & 0,4 & $0,4-0,5$ \\
Revisión de pies & 4,7 & $3,9-5,5$ \\
Revisión de calzado & 4,3 & $3,6-5,0$ \\
Ausencia del hábito de & 6,6 & $5,5-7,8$ \\
fumar & 4,6 & $3,8-5,4$ \\
\hline Promedio &
\end{tabular}

El puntaje promedio fue 4,6, correspondiendo al $66 \%$ de la adherencia perfecta, cifra similar a lo que la American Association of Clinical Endocrinologist reportó en el período 2003-2004 (cerca del 70 \%) (28) y a lo que la Organización Mundial de la Salud (OMS) señaló como esperable para países en desarrollo en su informe sobre adherencia a tratamientos en enfermedades crónicas del 2004 (29).

Los puntajes más elevados se registraron para la ausencia del hábito de fumar $(6,6)$ y el cumplimiento de medicación, 6,2, comparable a lo encontrado por otros autores $(30,31)$; mientras que las peores puntuaciones se registraron en el automonitoreo de glucosa, la práctica de ejercicio físico y las actividades preventivas como cuidado de extremidades. 
La importancia de la práctica de ejercicio físico en el control de la diabetes está suficientemente demostrada al igual que su sinergia cuando se acompaña de dieta y control de peso corporal (32), sin embargo, la motivación para la realización de ejercicio físico en ocasiones obedece más a razones de estética que terapéuticas (33), pudiendo inclusive hallarse percepciones francamente negativas $(34,35)$.

En resumen, y coincidente con la evidencia, se reconoce que los elementos álgidos en el logro de niveles óptimos de adherencia lo constituyen la dieta y el ejercicio $(20,21,37,38)$; sin embargo, el estudio que aquí se presenta demuestra la necesidad de fortalecer también el automonitoreo y el cuidado de extremidades.

Control metabólico y adherencia al tratamiento

El control metabólico se puede determinar midiendo niveles de glucemia en ayunas o por medio de la cuantificación de la hemoglobina glucosilada A1C (HbA1C). Este último indicador ha sido considerado como el mejor para el pronóstico de complicaciones y como el que actualmente mejor da cuenta de los niveles próximos pasados de glucemias.

En el grupo de pacientes evaluados, el 58 \% alcanzó las metas de HbA1C menor o igual que 6,5 \%, consideradas como adecuado pues indican niveles glucémicos cercanos a los valores normales; el 24 \% registró control aceptable (HbA1C entre 6,5 y $8 \%$ ), y el $18 \%$ mostró niveles no aceptables, con cifras superiores al $8 \%$. De manera similar, un estudio realizado en la ciudad de Medellín, Colombia, determinó que sólo el 43,6 \% de los pacientes diabéticos tipo 2 alcanzó cifras de HbA1C que no superan el límite de 7 \%, y puso en evidencia porcentajes preocupantes de prevalencia de complicaciones (39).

Clasificando a los pacientes según el puntaje que obtuvieron para adherencia con respecto al promedio $(4,6)$ y relacionándolos con los niveles de control metabólico, se observa la distribución que se presenta en la Figura 1.

Aunque las diferencias entre los grupos no fueron estadísticamente significativa $(p=0,12)$, resulta importante anotar que incluso peores adherencias corresponden a un ligero mejor control, situación comprensible si se toma en consideración la fuerte influencia de variables tales como edad, tiempo de diagnóstico y comorbilidades, así como lo múltiple y complejo de la relación adherencia/control 
Figura 1. Niveles de adherencia auto-reportada según el control metabólico logrado por los pacientes en estudio

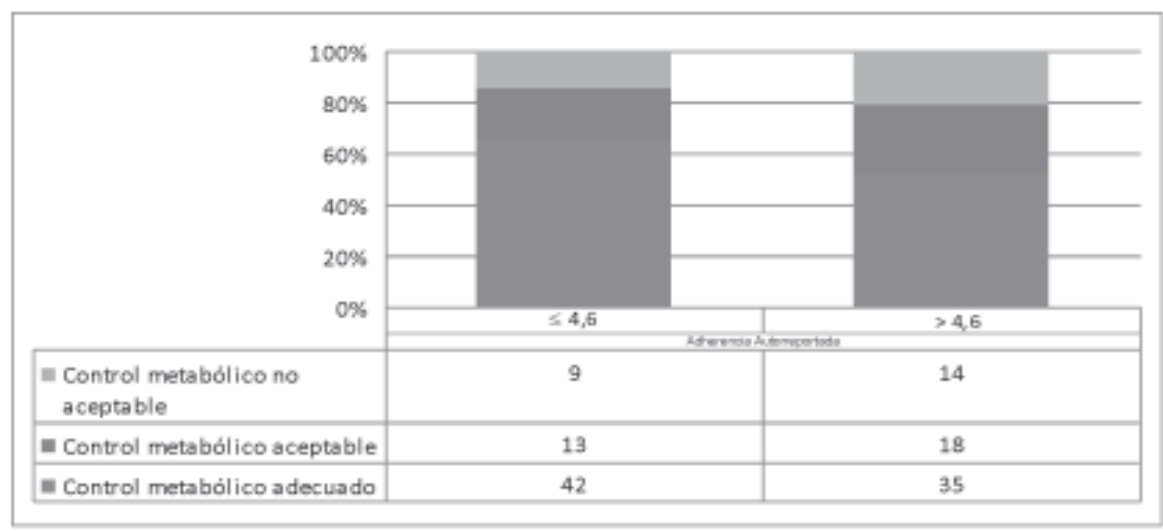

\section{DISCUSIÓN}

La adherencia al tratamiento en enfermedades crónicas es un tema multifactorial y complejo. En éste se mezclan factores psicológicos, demográficos y de comportamiento, así como aspectos propios debidos a la cronicidad de la afectación y otros que atañen al individuo como tal y como ser social y cultural.

A pesar de la enorme importancia que tiene y se le ha reconocido siempre al tratamiento farmacológico en el cuidado del paciente diabético, los resultados obtenidos en este estudio muestran la necesidad de fortalecer los pilares no farmacológicos. Las tasas más bajas de adherencia comprometen en mayor medida la actividad física y la dieta, específicamente el consumo de vegetales, así como las acciones preventivas de cuidado de miembros inferiores, en los cuales pudiera ser posible lograr grandes beneficios con mínima inversión, mediante refuerzos específicos desde los programas educativos.

Existen elementos, sin embargo, que poseen un fuerte componente económico que excede el deseo y la disposición del paciente hacia el cambio de comportamiento. En estos casos, la fuerza que facilite el cambio de comportamiento debe surgir de políticas públicas o privadas, que disminuyan la brecha de accesibilidad mejorando la oferta y precio de productos aptos para la dieta del diabético, tan escasos en la Región Caribe colombiana, abriendo más espacios de promoción de hábitos saludables e implementando más lugares habilitados y seguros para la realización de ejercicio físico. 
Los resultados medidos en términos de control metabólico son escasos, con efectos deletéreos tanto desde el punto de vista económico, por los recursos que se invierten, como desde el punto de vista psicosocial, generando mayores niveles de stress y depresión (34), existiendo la posibilidad de que adherencias parciales o incompletas estén pasando inadvertidas, debido a la falta de conocimiento o comprensión incorrecta de las acciones que se deben llevar a cabo (26).

Los cambios en los patrones de consumo y hábitos de vida, unidos a mayores expectativas de vida, permiten predecir un futuro en el cual las enfermedades crónicas serán las de mayor prevalencia (36), razón por la cual las implicaciones de los resultados de este estudio no se ubican únicamente en el ámbito de los programas educativos que brinda la EPS, sino que también deben considerarse para la organización de los programas que desarrollan los municipios, los distritos, el departamento y el país, con miras a poder alcanzar proporciones mundiales.

De acuerdo con los resultados obtenidos, las mayores dificultades se presentan en dieta y ejercicio; por tanto, entre las posibles soluciones, se sugiere implementar alianzas entre regiones para facilitar el intercambio de alimentos saludables a precios más accesibles, trabajar los entornos urbanos para propiciar el ejercicio, adecuando escenarios deportivos institucionales y públicos y promoviendo alimentación saludable.

Por lo expresado anteriormente, y con el propósito de diseñar estrategias de mayor cobertura e impacto, es importante realizar estudios regionales que permitan extraer conclusiones con mayor poder de generalización y complementar la mirada psicosocial que se ha presentado en este trabajo, que presenten una visión desde las culturas, las instituciones y la política pública de tal modo que permitan intervenciones mejor adaptadas a las características específicas del grupo al que van dirigidas.

En este escenario es donde mejor se dimensiona la necesidad de comenzar a desarrollar trabajos de investigación e intervenciones sinérgicas entre los distintos actores involucrados, que permitan cumplir con los objetivos de mejorar el control del padecimiento y lograr la prevención o retraso, en la medida de lo posible, de la aparición de las complicaciones propias de la enfermedad, con la consecuente repercusión en el impacto social y económico. Es impostergable, por tanto, tomar conciencia de que los perfiles de morbimortalidad del futuro dependerán, en gran medida, de las acciones que se tomen en el presente 
Agradecimientos. La investigación cuyos resultados se presentan en este artículo se realizó gracias al apoyo financiero del Instituto de Investigaciones Biomédicas de la Universidad de San Buenaventura. Los autores agradecen a las siguientes personas e instituciones: Dr. Javier Palacio, Director Laboratorio Génesis de Cartagena; Dr. Harold Bermúdez, asesor estadístico; Dra. Lourdes Benítez, Decana Facultad de Ciencias de la Salud de la Universidad de San Buenaventura de Cartagena; Drs. Rafael Tuesca y Edgar Navarro, docentes e investigadores de la Universidad del Norte. También presentan sus agradecimientos a la Dirección de Investigaciones y Proyectos de la Universidad del Norte, así como a los directivos y funcionarios de COOMEVA EPS Cartagena y los pacientes que participaron en este estudio

Conflictos de interés: Ninguno

\section{REFERENCIAS}

1. Alcaldía Mayor de Cartagena de Indias, Distrito Turístico y Capital, Departamento Administrativo Distrital de Salud (DADIS), Dirección Operativa de Salud Pública, Programa Vigilancia en Salud Pública. Comportamiento de los eventos bajo vigilancia epidemiológica 2006. [Internet]. Disponible en: http://www.dadiscartagena.gov.co/documentos-yarchivos/perfil-epidemiologico/3.html. Consultado Agosto del 2007.

2. Hsiao W. Colombian Health System Reform. Cambridge: School of Public Health. Harvard University; 1995.

3. Medici A., Londoño J., Coelho O and Saxenian H. Managed care and managed competition in Latin America. In: Schieb G. J., editor. Innovations in health care financing. Washington D.C: The World Bank; 1997. p. 215-231.

4. Departamento Administrativo Distrital de Salud (DADIS), Dirección Operativa de Aseguramiento 2007. [Internet]. Disponible en: http://www.dadiscartagena.gov.co/direccionoperativa-de-aseguramiento/index.php. Consultado Agosto del 2007.

5. República de Colombia. Ley 1122 de 2007(enero 9) por la cual se hacen algunas modificaciones en el Sistema General de Seguridad Social en Salud y se dictan otras disposiciones; 2007. [Internet]. Disponible en: http://www.asivamosensalud.org/descargas/ ley1122_2007.pdf.Consultado Febrero del 2008.

6. Selli L, Kauffmann L, Meneghel S, Zapico J. Técnicas educacionales en el tratamiento de la diabetes. Rev Saúde Pública 2005; 21(5):1366-1372.

7. King H, Aubert R, Herman W. Global Burden of Diabetes, 1995-2025 Prevalence, numerical estimates, and projections. Diabetes Care 1998; 21:1414-1431, 1998

8. Gobernación de Bolívar, Secretaría Seccional de Salud, Unidad de Salud Pública y PAB. Diagnóstico situacional de Salud, Bolívar; 2004.

9. Alayón A, Alvear C. Prevalencia de desórdenes del metabolismo de los glúcidos y perfil del diabético en Cartagena de Indias, Colombia, 2005. Revista de la División de Ciencias de la Salud de la Universidad del Norte 2006; 22(1): 20-28.

10. Alcaldía Mayor de Cartagena de Indias, Distrito Turístico y Capital. Departamento Administrativo Distrital de Salud-Subdirección de Salud Pública, Oficina de Vigilancia en Salud Pública. Perfil epidemiológico del distrito de Cartagena; 2004.

11. Alcaldía Mayor de Cartagena de Indias y Departamento Administrativo Distrital de Salud (DADIS). Programa para la Promoción y Prevención de las Enfermedades Crónicas no Transmisibles del Distrito de Cartagena; 2006.

12. Alpízar M. Guía para el manejo integral del paciente diabético. México: El Manual Moderno; 
786 REVISTADE SALUD PÚBLICA·Volumen 10 (5), Diciembre 2008

2001.

13. Egede L. Lifestyle modification to improve blood pressure control in individuals with diabetes is physician advice effective? Diabetes Care 2003; 26:602-607.

14. Laaksonen D, Lindstro"m J, Lakka T, Eriksson J, Niskanen L, Wikstro"m K, Aunola S, Keina"nenKiukaanniemi S, Laakso M, Valle T. Physical activity in the prevention of type 2 diabetes the finnish diabetes prevention study group diabetes 2005; 54:158-165.

15. Lutfey K, Wishner W. Beyond "compliance" is "adherence" improving the prospect of diabetes care. Diabetes Care 1999; 22:635-639.

16. Schwartzmann L. Calidad de vida relacionada con la salud: Aspectos conceptuales. Cienc. enferm 2003; 9(2) [Internet]. Disponible en: http://www.scielo.cl/ scielo.php?script=sci_arttext\&pid=S0717 95532003000200002\&lng=es\&nrm=iso>. ISSN 0717-9553. Consultado Diciembre del 2006.

17. Federation Internacional Pharmaceutical-FIP. Declaración de las normas profesionales de FIP: El papel del farmacéutico en la estimulación de la adherencia a los tratamientos a largo plazo. Approved by FIP Council in Sydney in September 2003.

18. Clark N, Becker M. Theoretical models and strategies for improving adherence and disease management. In: Shumaker S., editor. The handbook of health behavior change. Estados Unidos: Springer Publishing Company; 1998.

19. Grant R, Devita N, Singer D, Meigs J. Polypharmacy and medication adherence in patients with type 2 diabetes. Diabetes Care 2003; 26:1408-1412.

20. Hernández-Ronquillo L, Téllez-Zenteno J, Garduño-Espinosa J, González-Acevez E. Frecuencia y factores asociados al incumplimiento terapéutico en pacientes con diabetes mellitus tipo 2. Salud Pública Mex 2003; 45:191-197.

21. Nelson K, Reiber G, Boyko E. Diet and exercise among adults with type 2 diabetes findings from the third national health and nutrition examination survey (NHANES III). Diabetes Care 2002; 25:1722-1728.

22. American Diabetes Association. Economic costs of diabetes in the U.S. in 2002. Diabetes Care 2003; 26(3): 917-932.

23. Mata M, Antoñanzas F, Tafalla M, Sanz P. El coste de la diabetes tipo 2 en España. El estudio CODE-2. España. Gaceta Sanitaria 2002; 16(6): 511-520.

24. Menéndez J, Guevara A, Arcia N, León Díaz EM, Marín C, Alfonso JC. Enfermedades crónicas y limitación funcional en adultos mayores: Estudio comparativo en siete ciudades de América Latina y el Caribe. Rev Panam Salud Pública 2005; 17(5/6):35361.

25. Caporale J, Calvo H, Gagliardino J. Costos de atención médica de personas con diabetes anteriores y posteriores a su hospitalización en Argentina. Rev Panam Salud Pública. [Internet]. 2006 Dic; 20(6):361-368. Disponible en: http://www.scielosp.org/ scielo.php?script=sci_arttext\&pid=S1020-49892006001100001\&lng=es\&nrm=iso. Consultado Julio del 2006.

26. Toobert D, Hampson S, Glasgow R. The summary of diabetes self-care activities measure: Results from 7 studies and a revised scale. Diabetes Care 2000. 23(7): 943-950.

27. OMS. Adherencia a los tratamientos a largo plazo: Pruebas para la acción 2004. [Internet]. Disponible en: http://www.paho.org/Spanish/AD/DPC/NC/nc-adherencia.html Consultado Enero del 2007).

28. Asociación Americana de Endocrinólogos Clínicos-AACE. Directrices médicas para el tratamiento de la diabetes mellitus: El sistema AACE de autotratamiento intensivo de la diabetes-actualización 2002. Endoc Pract 2005, 8 (Suplemento 1) 40-82. [Internet]. Disponible en: http://www.aace.com/pub/pdf/guidelines/diabetes_2002.pdf. Consultado Enero del 2007.

29. Martín L. Repercusiones para la salud pública de la adherencia terapéutica deficiente. Rev Cubana Salud Pública . jul.-set. 2006; 32(3) p.0-0. [Internet]. Disponible en: http://scielo.sld.cu/scielo.php?script=sci_arttext\&pid=S0864-34662006000300013\& Ing=pt\&nrm=iso > Consultado Julio del 2007. 
30. Cramer J. A systematic review of adherence with medications for diabetes. Diabetes Care 2004; 27:1218-1224.

31. Mc Nabb W. Adherence in diabetes: Can we define it and can we measure it? Diabetes Care. Alexandria 1997; 20(2):215-218.

32. Hu FB, Manson JE, Stampfer MJ, et al. Diet, lifestyle, and the risk of type 2 diabetes mellitus in women. N Engl J Med 200; 345:790-797.

33. Martínez E. La Salud como motivación para la práctica de actividad física en personas adulta. latreia 2003; 16(1):32-38.

34. Institute of Medicine Staff. Health and behavior: The interplay of biological, behavioral, and societal influences. Washington, DC USA: National Academies Press, 2 2002. [Internet]. Disponible en: http://site.ebrary.com/lib/unorte/Doc?id=10032404\&ppg=20 Consultado Abril del 2006.

35. García R, Suárez R. Resultados de un seguimiento educativo a personas con diabetes mellitus tipo 2 y sobrepeso u obesidad. Rev Cubana Endocrinol 2003; 14(3). Disponible en: http://www.bvs.sld.cu/revistas/end/vol14_3_03/end04303.htm Consultado Abril de 2006.

36. Roales-Nieto J, Moreno E. Hábitos básicos de salud y creencias sobre salud y enfermedad en adolescentes de España, Colombia y México. Revista Latinoamericana de Psicología 2004; 36(3): 483-504.

37. Jacobsen D, Donelly J, Snyder-Heelan, Livingston K. Adherence and attrition with intermittent and continuous exercise in overweight women Int. J. Sports Med 2004; 24:459-464.

38. Jáuregui J, de la Torre A, Gómez G. Control del padecimiento en pacientes con diabetes tipo 2 e hipertensión arterial: Impacto de un programa multidisciplinario. Rev Med IMSS 2002; 40 (4): 307-318.

39. Villegas Perrasse A, Abad SB, Faciolince S, Hernández N, Maya C, Parra L. El control de la diabetes mellitus y sus complicaciones en Medellín, Colombia, 2001-2003. Rev Panam Salud Pública 2006; 20(6):393-402 